\title{
Gender medicine: an up-date
}

\author{
Cecilia Politi, ${ }^{1}$ Tiziana Ciarambino, ${ }^{1}$ Flavia Franconi, ${ }^{2}$ Lucrezia De Feudis, ${ }^{3}$ Maria Carolina Mayer,${ }^{4}$ \\ Alessandra Martignoni, ${ }^{5}$ Ilaria Giovi, ${ }^{5}$ Michela Tonani, ${ }^{5}$ Salvatore La Carrubba,,${ }^{6}$ Anna Maria Moretti, \\ Carmen Martino, ${ }^{7}$ Giovanni Scanelli ${ }^{8}$
}

${ }^{1}$ UOC Medicina Interna, Isernia; ${ }^{2}$ Dipartimento di Scienze Biomediche, Università di Sassari; ${ }^{3}$ UOC Medicina Interna Ospedale S. Spirito, Pescara; ${ }^{4}$ Medicina Interna ad indirizzo endocrinologico, Ospedale AORN “A. Cardarelli”, Napoli; ${ }^{5}$ Stroke Unit, Fondazione IRCCS Policlinico San Matteo, Pavia; ${ }^{6}$ UOC Medicina Interna, Ospedali Riuniti di Palermo "Villa Sofia", Palermo; ${ }^{7}$ UO Malattie Apparato Respiratorio, Azienda Ospedaliera-Universitaria Policlinico Bari; ${ }^{8}$ Dipartimento Medico, AOU “Arcispedale Sant'Anna”, Ferrara, Italy

\section{ABSTRACT}

Women get sick more, use more health services, take more drugs, and have a higher frequency of serious adverse reactions. Despite this, the drugs we use are little studied in women: both clinical studies and pre-clinical experiments are carried out predominantly in males and the young. Before 1990 , no more than $26-30 \%$ of women were usually enrolled in a trial, so we lacked the statistical power of showing the efficacy and safety of the drugs being studied in the whole population. The gender blindness (transposition of the male studies in female populations) resulted in a lack of awareness of the differences between males and females, and this prevented both genders from receiving the best possible care. This gender bias also, to a lower degree, hurts men: depression, migraines, osteoporosis have not been studied properly in males. Although the process is slow, the scientific community has begun to pay more attention to direct and indirect influences that gender exercise on biological mechanisms, and this includes both internal and external cultural and environmental factors. Therefore, the differences between the old, the young, children, and pregnant women (considered the third gender group) will become increasingly more important as care becomes more personalized. The first course of gender medicine was established only in 2002 at Columbia University, New York, USA. The World Health Organization has incorporated gender medicine into the Equity Act. This implies that the treatment given must be the most appropriate and best suited the individual patient's gender. The Committee on Women's Health of the Ministry of Health in Italy was established in 2007. Institutions now pay great attention to the importance of this clinical perspective and are sensitive to the need for change. This review focuses on specific open questions regarding gender: pharmacology, clinical trial recruitment, cardiovascular prevention, stroke, osteoporosis, chronic obstructive pulmonary disease, depression, and discusses presentations made to the $1^{\text {st }}$ course of gender medicine organized as part of the $18^{\text {th }}$ National Congress of the Federation of Associations of Hospital Doctors on Internal Medicine (FADOI), 2012.
\end{abstract}

Correspondence: Cecilia Politi, UOC Medicina Interna, Ospedale "F. Veneziale", Isernia, Italy.

Tel. +39 0865442306. E-mail: cecilia.politi@asrem.org

Key words: gender medicine, pharmacology, clinical trials, cardiovascular prevention, stroke, chronic obstructive pulmonary disease, depression, osteoporosis.

Contributions: CP, TC, draft of paragraph Gender medicine and sub-paragraphs, manuscript preparation and supervision; FF, draft of paragraph Gender medicine and gender pharmacology; LD-F, draft of paragraph Women in large trials: the blind side; MCM, draft of paragraph Cardiovascular prevention in women; AM, IG, MT, draft of paragraph Stroke and women; SL-C, draft of paragraph Gender and depression; AMM, CM, draft of paragraph Chronic obstructive pulmonary disease and women; GS, draft of paragraph Men and osteoporosis.

This work is licensed under a Creative Commons Attribution NonCommercial 3.0 License (CC BY-NC 3.0).

CCopyright C. Politi et al., 2013

Licensee PAGEPress, Italy

Italian Journal of Medicine 2013; 7:96-106

doi:10.4081/itjm.2013.96

\section{Gender medicine}

\section{Introduction}

Gender medicine (GM), a recent branch of the biomedical sciences, was founded to ensure everyone (regardless of gender) receives the best possible care. GM is not a mere scientific speculation; it is an essential tool for our National Health System (NHS).

With the term sex we define a difference closely linked to biology, while with the word gender we define the differences linked both to social culture and to the environment. We all understand how the environment (both internal and external factors) can modify hereditary characteristics. Therefore, we need to take into consideration numerous different elements that co-operate and interact within a much more complex system in which diversity is not only a source of conflict but also of enrichment. This conditions adaptation (to external stimuli), vulnerability, but also a different pharmacological response. ${ }^{1}$

Furthermore, we must recognize that we have a poor knowledge of how gender influences health. 


\section{Gender blindness}

Simply widening to both sexes the knowledge obtained from studies conducted primarily or exclusively on male animals or men led to the so-called gender blindness. This implies the failure to identify differences in large fields of medicine.

If a drug or a medical-surgical device is not specifically tested on a given population, there is no way of knowing what the real safety and efficacy conditions on this population will be. ${ }^{1}$

This limits the excellence of research and hence the potential benefit to society.

Thanks to advances in science, there has been a noticeable reduction in mortality rates of male patients. But rates in female patients are not as significant. In Italy, female cardiovascular and cerebrovascular mortality is approximately $50 \% .{ }^{2}$ Morbidity and mortality for chronic lung disease (mostly affecting males) are increasing, as well as female disability (twice that of men). Moreover, the screening campaigns are sexoriented on reproductive organs (bikini medicine), with little attention to the metabolic syndrome, treatment and prevention of obesity, or encouraging women to stop smoking. Adverse drug reactions in women are more frequent and more severe in terms of mortality, hospitalization and longer hospital stays. In transplant settings, gender differences are also detectable: survival rates change according to donor-recipient gender combination. Approximately $60 \%$ of donated organs go to males: a woman with end stage renal disease is $25 \%$ less likely to receive a kidney than a man. ${ }^{3}$ But let us look at other diseases such as depression which in males is diagnosed less but with an extremely high successful suicide rate, and osteoporosis usually considered a problem of the elderly women, but extremely disabling in men.

There are also other gender factors: the child is considered and treated as a little man (very few trials are conducted on children and in pediatric oncology drug use is largely off-label), the pregnant woman (the $3^{\text {rd }}$ gender), the woman who uses contraceptives: the other woman (editor's note: fertile women are regularly excluded from clinical trials) and the elderly.

\section{Gender medicine: today and tomorrow}

There is great interest in GM among both the international scientific community and on an institutional level. In 2000, the World Health Organization (WHO) included GM in the Equity Gender Act. This stated that treatment be more appropriate and more suited to each gender. In Italy, in 2007, Senator Livia Turco included GM in the Women's Health Committee, to create the universalism of health (art. 32 of the Italian Constitution) more effective and be able to support gender research (e.g. authorization of new drugs in which gender had been equally represented). The $1^{\text {st }}$ course in gender medicine (A new approach to health care based on insights into biological differences between women and men) started in 2002 at Columbia University, New York, USA, and since then many others have been set up in Europe. The number of GM articles published in PubMed has tripled in the last years. In 2011, the Italian Agency for Pharmaceuticals (AIFA) established the Working Group on Drugs and Gender.

At the Health Higher Institute (ISS), a Degenerative Ageing Illnesses and Gender Medicine ward has been set up within the pharmaceutical department. This has a wide range of research tasks: biomedical, translational (cancer, drugs, with pathogenic studies on associated gender biomarkers, on the differences in response in the oncological field linked to gender, on safety at work and gender), and with regulatory activities and checks, especially in phase I drug trials.

GM should also be considered a necessity for excellence in both basic and clinical research through the acquisition of new knowledge. But a gender approach is also essential to address health policy that responds to the fundamental principle of our NHS of the equity and appropriateness of care.

In an era of phenotypic medicine, we are not practicing evidence-based medicine in a relevant part of the population treated. GM must, therefore, become a key part of medical education with gender culture integrated into the curriculum of all health professions, both scientific and technical.

The Italian hospital internal medicine community has accommodated all these needs with the $1^{\text {st }}$ course of gender medicine organized by the National Congress of the Federation of Associations of Hospital Doctors on Internal Medicine (FADOI) in 2012, so that knowledge of the differences between different populations (elderly, adolescent, woman) have a greater impact on the quality and on the personalization of care, reducing the level of errors and generating savings for the NHS. This course focuses on specific open questions regarding gender: pharmacology, clinical trial recruitment, cardiovascular prevention, stroke, osteoporosis, chronic obstructive pulmonary disease (COPD), and depression.

\section{Gender medicine and gender pharmacology}

'Of all the forms of inequality, injustice in health care is the most shocking and inhumane.' Martin Luther King

(in a speech to the $2^{\text {nd }}$ National Convention of the Medical Committee for Human Rights, 25 March 1966, Chicago, IL, USA)

The biological differences (sex) and the sociocultural ones (gender) have an impact on the health of 
men and women. So the time has come to abandon the view that the human male is the norm because this has caused numerous disadvantages women's health leading to the application of a less evidence-based medicine. It is opportune to remember that in some areas (osteoporosis, depression, etc.) males are disadvantaged. Therefore, it is necessary to overcome this gender gap to reach equity of treatment through the inclusion of a gender approach both in pre-clinical and clinical studies adopting the biosocial model of illness. Adoption of this biosocial model appears to be of fundamental importance considering that women very often occupy a lesser social role and so are economically poorer. The inverse relationship between poverty and illness is well known and this could explain the so-called female paradox: women live longer but become ill more than men. The majority of women also has the role of caregiver and is the shock-absorber of the family. This causes a great deal of stress with a consequent increase in the risk of numerous pathologies, not only of a psychiatric nature. It worth remembering that men and women respond differently to stress, and in women the response is also modulated by the phases of life through sex hormones. Furthermore, the role of caregiver implies less free time that causes greater difficulty in carrying out regular physical activity, encouraging the insurgence of obesity with all its consequences. Another very important factor is violence since, in Italy, almost seven million women between the ages of 16 and 70 are victims of violence. Between the ages of 16 and 44, violence kills more women than cancer and road accidents. If the immediate consequences of violence are well known, less known are the delayed consequences that go far beyond the psychiatric consequences that are potentially devastating also for the daughters and sons.

Lastly, a disadvantage for women is highlighted in the field of pharmaceutical treatment both for representative inappropriateness or underrepresentation of women. ${ }^{1}$ There is also poor consideration for women's hormonal variations that can alter the pharmacological response and for her various life phases (e.g. pregnancy). Despite women being smaller than men, the dose for pharmaceuticals is generally measured on men (body weight $70 \mathrm{~kg}$ ). In addition to the difference in size, there are other differences, such as differences in corporeal composition, in metabolism, different elimination rates and processes that determine variations in the pharmacokinetic parameters. ${ }^{4,5}$ Furthermore, pregnant women or those who take combined oral estrogen-progestin association can have their own pharmacokinetic characteristics. Today, we know that there are also important pharmacodynamic differences. The poor attention given to women in pharmacological studies is reflected in their feelings of diminished security. In fact, adverse reactions are more common in women than in men. Furthermore, they are more serious in women, causing a greater number of hospital admissions.

It is clear that if we wish to take steps towards an evidence-based medicine and towards a personalized approach the first step is to give voice to gender medicine. In addition, the application of sex-gender medicine leads, according to the World Bank, to a more rapid economic growth of the territory, which is not to be taken lightly in a period of crisis. Given this, we are starting to consider gender issues in medicine.

\section{Women in large trials: the blind side}

'The world was made for men and not for women.' Oscar Wilde

(Lady Stutfield in the play: A woman of no importance - ACT I, 19 April 1893, Theatre Royal Haymarket, London, UK)

The largest and most important clinical trials carried out in the recent past were designed by men and carried out on men. Traditionally, women were omitted or underrepresented. The presence of the female gender in the intervention studies of primary cardiovascular prevention such as The Physicians' Health Study, The Multiple Risk Factor Intervention Trial and The Baltimore Longitudinal Study, is practically nonexistent since these clinical trials were strictly centered on the male gender. Several reasons may explain this: i) a form of protection towards the female gender or to a human body whose predominant function was considered to be procreation; ii) the role of caregiver considered typical of women; iii) the complexity and the costs of a clinical trial that had to consider the female physiological hormonal variations; and iv) the high mortality rate observed in males in relation to age.

In fact, for many years, in clinical medicine, a typically phallocentric attitude has prevailed; in the cultural patriarchal tradition men represent logos. The interest towards women has mainly centered upon her reproductive organs, and the possibility that they could fall sick and die for other reasons has been seriously underestimated. Diagnostic procedures, precautionary measures and treatment of proven efficacy in men were applied to women without any modification with the implicit assumption that the male was representative of both genders.

At the beginning of the 1990s, in the United States, progressively more and more women were being included in clinical trials thanks to statutory regulations of the National Institutes of Health (unfortunately, such regulations have still not been established in Europe). In fact, there was a heightened awareness on the part of the scientific community that breast cancer is not the main and only cause of death. This change in attitude was also a result of the growing female pres- 
ence in the medical profession. To date, though, clinical trials still suffer from a lack of female participation and are, therefore, inadequate because of the clinical epidemiology examined and for the potential gender differences.

A recent analysis of randomized clinical trials that involve pharmaceutical drugs for cardiovascular diseases, carried out in Europe in the years 2006-2009 demonstrate that women represent only one-third of the entire population studied and that data involving the difference induced by gender are reported only in $50 \%$ of cases. ${ }^{6}$

The inclusion of women in clinical studies does not represent simply the latest request of the feminist, but is a real necessity to guarantee effective, safe and appropriate treatment in clinical practice also for the female gender. In fact, it is legitimate to only translate the evidence from research into clinical practice on the condition that the population belonging to the female gender is adequately represented in the studies. Data derived from sub-tests, post hoc tests and from studies that do not consider the impact of gender in the population studied can be statistically inappropriate and/or confusing in their conclusions. For example, is the efficacy of the ASA in the prevention of stroke in women a statistical artifact or a real example of gender difference? ${ }^{7}$

Women and men have specific qualities and it would be reductive to consider them as a single biological group.

\section{Cardiovascular prevention in women}

\section{Epidemiology}

In the industrialized world, and in many emerging countries, heart problems represent the most frequent cause of death in women and account for approximately one-third of all cases of female deaths. Contrary to what is commonly thought, more women than men die from cardiovascular illnesses and myocardial infarction is more often fatal in women. The wellknown tendency to reduce mortality caused by coronary artery disease (CAD) in the last 15 years has concerned men more than women, further increasing the divide between the two genders. In particular, CAD is clearly increasing in women of childbearing age (35-54 years old) probably due to the increase in obesity and the fact that so many women still smoke. ${ }^{8}$

\section{Physiopathology}

Female CAD presents several particular characteristics and, in particular, it is quite frequent to find an almost normal coronary tree after a heart attack, to the point that recently several authors ${ }^{9}$ proposed to define the female experience ischemic heart disease instead of CAD, a term until now commonly used in Englishspeaking countries and that should be reserved for the illness in males.

In women, the continuous fluctuation of hormones through life determines a microvascular and endothelial malfunction, with small coronary plaques that are not obstructive but very unstable, and with a tendency to cause emboli, i.e. microvascular angina. The response to the primitive lesion of the intima would, therefore, be different in men and in women, with regard to its remodeling mechanisms and to vascular reactivity.

\section{Prevention}

An increase in awareness of the risk of female cardiovascular illness is absolutely essential. Up till now, there has been a tendency among doctors to delay adoption of preventive measures until women have reached menopause. But women themselves also need to change their approach so that they can put into practice the recommended measures correctly. Unlike that of a man, the life of a woman is marked by physical events linked to time (first menstruation, monthly cycle, pregnancies, menopause). Since many of these events, and in particular pregnancy, put women in contact with doctors it is opportune to take advantage of their involvement and start the appropriate preventive measures. According to the latest guidelines on prevention, ${ }^{8}$ women can be stratified into three classes of risk: women at high risk, women at risk, women with an ideal cardiovascular situation. The recommendations, based on evidence from specific studies on women, do not differ substantially from those for men: i) intervention in lifestyle: cigarette smoke, eating habits, fatty acids, omega-three, exercise, maintenance or reduction of body weight; ii) pharmacological and non-pharmacological intervention in risk factors (arterial pressure, diabetes, dyslipidemia) and preventative pharmacological intervention (antiplatelets, anticoagulants, $\beta$-blockers, ACE-inhibitors, sartans, anti-aldosterones) applied with different intensity according to the class of risk.

The only substantial differences concern two class III recommendations (these are interventions that are useless, inefficient and potentially dangerous). It is not recommended that women under the age of 65 years to use aspirin as a routine for primary prevention. In addition, for primary and secondary prophylaxis neither antioxidant vitamin supplements nor hormone replacement therapy (HRT) should be used. Nor should selective estrogen receptor modulators be used for women who have reached menopause.

This last recommendation contrasts with the guidelines issued at the end of the last century but agrees with the results of the last clinical studies that have demonstrated an unfavorable risk-benefit profile for HRT. ${ }^{10}$ 


\section{Stroke and women}

\section{Overview of epidemiological features of cerebrum vascular disease in women}

The clinical relevance of stroke is increasing in the female gender. It affects women of all age groups and women affected from stroke at an the older age overtake men both in terms of prevalence and incidence. ${ }^{11,12}$ Stroke is the leading cause of disability and has many socio-sanitary implications for women. For example, after a first event, women are hospitalized more frequently than men $(7.9 \%$ vs 3.9\%). As shown in an Italian study, ${ }^{13}$ this situation is probably related to the particular role of women in Italian families. Cardiac and cerebrum-vascular diseases are the first cause of women's death in Western society and their incidence is progressively increasing both in immigrants coming from South America and in the developing countries. ${ }^{14}$ Elderly women affected by the first stroke are on average five years older than men, they are affected by a more severe stroke, and have a worse outcome. In spite of the epidemiological data, health professionals and women themselves do not perceive stroke or the other cardiovascular diseases as a real danger. Women are certainly more afraid of other illnesses, such as breast cancer, than stroke.

\section{Clinical characteristic of stroke in women}

Many studies document that women with stroke call the Emergency Unit later than men and, once in hospital, are subjected to less diagnostic tests or therapeutically advanced procedures, e.g. intravenous and intra-arterial thrombolysis or carotid endarterectomy. Some authors consider this situation to be due to less awareness of disease by women. This could be related to women's social isolation (often elderly women are the only survivors in couples of advanced age) or to their economic status. Both of these conditions could be responsible for the absence of witnesses to the event and this increases the delay in calling for help. Some reports found many differences between women and men in terms of presenting symptoms and these explain why stroke in women is not immediately identified or, at worst, not identified at all. In fact, almost $43 \%$ of strokes in women present with problems in their state of consciousness, unspecific malaise, pain and headache, vertigo and imbalances. On the other hand, through a careful examination of the patient's case history and investigating events in the previous $24 \mathrm{~h}$, at least one prodromal and specific symptom can be identified. The symptoms described above are called atypical, and are less predictive and less specific in this illness compared to those traditional symptoms, such as hemiplegia, hemi-sensory deficit, disorders of speech or gait, sudden onset of headache, ataxia or impaired vision. ${ }^{15}$

\section{Pathogenic mechanisms of ischemic stroke in women of different ages}

Studies show an undertreatment of women in terms of primary and/or secondary prevention for cardiovascular diseases, and nowadays this can no longer be justified. They assume less statins or antithrombotic drugs, despite a higher incidence of atrial fibrillation in women older the age of 65 years, which is certainly a strong cause of cardio-embolic stroke. Almost 50\% of women of that age suffer from metabolic syndrome: the more the syndrome is complete (3-5 components present, Adult Treatment Panel III diagnostic criteria) the more it enhances the risk of transient ischemic attack/stroke, which becomes approximately double when compared to men. The metabolic syndrome is associated with early subclinical atherosclerosis, represented by left ventricular hypertrophy and carotid wall thickness (expressed as intimal media thickness). Arterial hypertension and atrial fibrillation are the most frequent risk factors for stroke in women.

Young people have strokes due to rare etiologies. These are generally more common in women since they are associated with arthritis, congenital or acquired thrombophilic conditions (pregnancy, peripuerperium), and predisposing conditions like migraine with aura, smoking, use of oral contraceptives, presence of patent foramen ovale, and atrial septal aneurysm with right to left cardiac shunt. Women are also at increased cardiovascular risk during the period of menopausal transition (from 40 to 59 years of age). This has, up to now been considered a period of low cardiovascular risk. In recent decades, it has been observed that women are losing their traditional advantage on men. In addition to traditional risk factors, other emerging factors, such as polymerase chain reaction, hyperomocysteinemia and its mutations, $\mathrm{Lp}(\mathrm{a})$, pro-inflammatory and pro-thrombotic factors (probably hormone-related) are also observed. Their effects are performed through metabolic mechanisms such as increased visceral adiposity, insulin resistance, increase in triglycerides and small dense low-density lipoprotein, reduced high-density lipoprotein. Also vascular mechanisms are involved: reduced endothelial function, increased oxidative stress, shift to a proinflammatory and prothrombotic state, which taken together can promote atherosclerosis with severe embolic risk, represented by the vulnerable plaque. ${ }^{16}$

\section{The future}

We do not really know if the difference between male and female gender of awareness and impact of stroke, of diagnostic workup, therapeutic process and prognosis can be explained by the different clinical presentation. It is also still a matter of interest whether the mere longevity of women justifies the above- 
mentioned differences but nowadays there is no definitive answer to these questions. It is clear that there is a need to develop health education campaigns to reinforce the information available to citizens and health professionals, in order to encourage rapid access to the Emergency Unit, and to treat women of all age groups in an appropriate way. Specific attention should be paid to the prevention of traditional cardiovascular risk factors (smoking, obesity, dyslipidemia, hypertension) and of those factors related to specific conditions in different age groups, such as pregnancy, puerperium, and menopause. This could be achieved, for example, through targeted collaborative initiatives between internists and gynecologists. We know that women have a major risk of bleeding with antithrombotic drugs, but there is no evidence-based reason why we should not adequately treat hypertension, atrial fibrillation, metabolic syndrome and dyslipidemia. We have to plan initiatives to promote a big change in lifestyle. However, most of the clinical trials carried out so far related to cardiovascular and cerebrum vascular diseases have excluded women or have included only small numbers of them.

There is, therefore, a great need for clinical trials providing gender-specific results and for studies on gender pharmacology, and health care professionals and scientific societies are called to participate in these. The aim should be to reduce the burden of cerebrum vascular diseases on women and to assure a fair health service.

\section{Gender and depression}

The overall prevalence of depressive symptoms in the community has been estimated to be $5-10 \%$, also in association with other comorbidities. ${ }^{17,18}$ Moreover, depression is highly underestimated and many people do not go to a psychiatric specialist. Consequently, depressive symptoms are common but, due to clinical and social reasons, are frequently undiagnosed and untreated.

Clinically, the diagnosis of depression is based on Diagnostic and Statistical Manual of Mental Disorders (DSM-IV) criteria, used mostly in psychiatric medicine. ${ }^{19}$ Epidemiological data showed that depression is 3:1 times more frequent in women than in men. In Italy, out of 8 million of patients potentially affected by depression, only 2 million are men and more than 5.5 million are women.

Data from a large survey carried out on patients admitted to Internal Medicine departments in Sicily showed a high prevalence of depressive symptoms: nearly $50 \%$ had mild symptoms, $13 \%$ had signs of severe depression of whom only $15.4 \%$ were already diagnosed as depressed and treated. According to previous studies, depressive symptoms were significantly more frequent in women than in men. ${ }^{20}$

\section{Clinical aspects in men and women}

In general, some clinical manifestations are similar in women and men, such as general sadness, pessimism, and sense of guilt. But men and women report it differently. In general, women more frequently say I am sad or I want to cry. Men affected by depression more frequently declare themselves to be anxious or tired, or report somatic symptoms such as headache.

Women's depressive symptoms are more frequently associated with more severe manifestations and are often related to specific moments, such as pregnancy or post-partum. In men, depression is characterized by motor inhibition. ${ }^{21}$

Tendency to suicide is more frequent in women. However, successful suicides are more frequent in men, probably due to the different ways in which the two genders attempt suicide: women more take a drug overdose or try to cut their veins, while men more frequently hang themselves or use firearms.

\section{Etiopathogenesis}

In 1915, Freud, in his theory of complementary series, affirmed that experiences in childhood, interacting with constitutional genetic factors lead to a natural predisposition to depression. In adulthood, traumatic factors could lead to clear psychiatric disease.

Recent studies confirm that genetic expression can be modulated by external experiences. This mechanism is mediated by modulation of gene expressivity, activating or inhibiting gene transcription and producing proteins that can determinate changes in cerebral functions.

On the other hand, it has been observed that increased volume of amygdala and reduced dimensions of the hippocampus are associated with the presence of depressive symptoms. Moreover, stress and glucocorticords are associated with reduced levels of brain derived neurotrophic factor (BDNF). Those modifications are influenced by antidepressants that can re-establish normal values of production of BDNF and dimensions of structures of the limbic system. These findings are in agreement with Freud's theories that consider a mental disease as results of genetic, physical and social factors.

Women are more exposed to biological and psychosocial factors. Biological factors are hormonal fluctuation in puberty, menstruation, pregnancy, postpartum, nursing, pre-menopause while psychosocial factors are double work load within and outside the home, burn out in taking care of others, such as children, less social recognition, violence in the family. Men are less exposed to hormonal fluctuations but psychosocial factors are significantly more important. In particular, these are related to a loss of status due to lack of social success at work, during period of rest 
from work, disease with consequent loss of ability, and diseases (hyperthyroidism or hypersurrenalism) or iatrogenic causes (hypertension, corticosteroids).

\section{Therapeutical approach}

In general, clinical trials on antidepressants always include men but less frequently include women. Women are often excluded because of potential confounding factors such as hormonal fluctuations, pregnancies, pre-menopause, contraceptives, or hormone therapies. But at same time, women are more frequently treated with antidepressants than men. This all results in a gap in our knowledge of the complete effects of these drugs on women and their interactions with other therapies.

The most frequently used drugs are first generation antidepressants (trycyclic), selective serotonin reuptake inhibitor, drugs with the combined action of serotonin and noradrenalin. The prescription of these drugs should take account of pharmacokinetics and pharmacodynamics, concomitant use of contraceptives, hormonal fluctuations, pregnancy, post-partum, nursing, pre-menopause and collateral effects such as weight gain and problems of libido. It must also be remembered that there are not sufficient data available regarding the different effects on women and men of new drugs on the market.

Neuroimaging demonstrated the efficacy of the pharmacological approach but also the usefulness of psychotherapy. Different mechanisms are involved but work in the same way. Antidepressant drugs are active from the limbic area to the cortex (going down), while psychotherapy is effective with action from the cortex to the limbic area (going up). Antidepressants produce effects quickly but once therapy is interrupted it is easier to lose efficacy. The efficacy of psychotherapy is slower but, interestingly, lasts significantly longer. ${ }^{22}$

\section{Chronic obstructive pulmonary disease and women}

The prevalence of COPD and related hospitalization in women are increasing. Recent years have also witnessed a major shift in the gender profile of the disease. Although earlier studies showed that men accounted for the majority of COPD-related deaths, the prevalence and mortality rate of COPD in women have more than doubled during the past 20 years in industrialized countries while they stabilized in men. ${ }^{23}$ The number of women dying of COPD in the United States and in Europe now surpasses that of men. ${ }^{23}$ In particular, over the past two decades, COPD-related mortality rates have also grown faster in women, and since the year 2000 more women than men have died from COPD. The greater prevalence of COPD and related mortality re- ported for men in earlier epidemiological studies may be due to underdiagnosis of women. Despite these differences, the international guidelines for the diagnosis and treatment of men or women with COPD are the same. It is important for healthcare professionals to recognize the gender differences in patients with COPD to optimize assessment, monitoring and treatment of this disease. In this regard, few studies have been performed to date in women.

Just how far these observations are valid is not known. Questions have been raised regarding how gender affects susceptibility to COPD and the following should be remembered: i) the number of women dying of COPD now surpasses men; ii) increased prevalence, mortality and variability of COPD in women versus men between 2002 and 2012; iii) factors such as prevalence of symptoms, triggering stimuli, response to treatment, susceptibility to smoking, frequency of exacerbations, impairment in quality of life response to oxygen therapy, presence of malnutrition, airway hyperresponsiveness and depression are all more frequently seen in women with COPD. Differences in age groups, prevalence of smoking, and criteria for defining airflow obstruction may partly explain the variations in prevalence between men and women.

Although increased tobacco use will contribute to the increasing prevalence of women with COPD, it has been hypothesized that women may be at greater risk of smoking-induced lung function impairment given the same level of tobacco exposure. The increase in prevalence and mortality for COPD among women compared with men is usually attributed to the delayed rise of smoking prevalence in women. ${ }^{24}$ It is also possible that sexual dimorphism of the human immune response may also be responsible for gender differences in the disease. More data are still needed on what the implications of these findings are on therapy and how gender influences the diagnosis, physiology, and presentation of COPD. ${ }^{25,26}$

Studies investigating gender differences in the vulnerability to the deleterious effects of tobacco either reported greater susceptibility to tobacco in women ${ }^{24}$ or failed to detect any sex disparities. ${ }^{24}$ Female smokers show greater bronchial responsiveness, ${ }^{27}$ lower baseline forced expiratory volume in $1 \mathrm{~s}$ (FEV1), and faster decline in FEV1, ${ }^{27,28}$ although this is not a hard-fast rule.

\section{Tobacco susceptibility}

As stated above, women may be at greater risk of smoking-induced lung function impairment given the same level of tobacco exposure. In Italy, $6.2 \%$ of women smoked versus $65 \%$ of men in 1957. Currently, $22.3 \%$ of young women smoke and $28.9 \%$ of men. ${ }^{24}$ However, mortality for women with COPD in the United States is also increasing. Between 1980 and 2000 mortality rates for COPD among men increased 
by $13 \%$ and have remained steady since 1985 . In women, however, between 1980 and 2000 death rates almost tripled, and in 2000 for the first time in terms of absolute numbers, more women than men died of COPD. ${ }^{26,28}$ Data from Canada also suggest increasing mortality for women with COPD. ${ }^{29}$ It remains unclear, however, whether biological differences between men and women have an impact on increasing mortality. Higher absolute mortality for COPD in women may be biased by the differential presence of sex hormones that appear to influence the metabolism of nicotine.

\section{Chronic exposure}

Several epidemiological studies have shown that chronic exposure to high levels of professional and non-professional agents (detergent, gas, latex) has adverse effects on respiratory health and influences chronic respiratory diseases. ${ }^{30}$ It has been reported that the exposure to these agents was $30 \%$ in men and 5$20 \%$ in woman.

\section{Diagnosis}

The effect of sex hormones on airway function is not clear. ${ }^{31}$ Sex hormones appear to influence airway function in asthma, but its contribution to COPD pathogenesis remains unclear. Future clinical trials in COPD should obtain data regarding factors associated with sex hormone exposure, including parity and history of oral contraceptive. There is a predominance of women using COPD contraceptives and hormone replacement therapy. Better epidemiological and mechanistic studies are required to better understand the immunological and hormonal determinants of fixed airway obstruction in non-smokers and how these relate to gender. ${ }^{24,32}$ Data suggest that chronic bronchitis is more common in women and emphysema more common in men. Other data demonstrated that women with emphysema were less likely to show reversibility of airway obstruction.

Detailed imaging studies in well-defined, large populations are required to define whether there are any gender differences in the manifestation of airway disease versus emphysema. ${ }^{26}$ In a general COPD population, the probability of respiratory deterioration when stopping inhaled steroids has been suggested to be higher in women than men. Difference in comorbidity between the genders should be addressed. This includes, among many others, differences in osteoporosis, depression and cardiovascular disease. However, further investigation is required to define whether autoimmunity is a more common phenomenon in women with COPD.

\section{Clinical}

Consistent with a previous report, women were four years younger than men when severe COPD was observed. An earlier development of COPD in women would imply the need for earlier diagnosis and treatment. Unfortunately, the diagnosis of COPD is often missed in women ${ }^{25}$ so that optimal treatment for this condition is delayed. Women had less severe airflow obstruction but more pronounced hyperinflation than men. While disease severity has traditionally been measured with FEV1, patient disability can vary greatly for a given level of obstruction, and measures of hyperinflation may give further relevant information on patient condition. ${ }^{28}$

COPD may evolve differently in men and women. Dissociation between the severity of airflow obstruction and of hyperinflation could occur if the pathological process is predominantly located at the small airway and/or parenchyma levels, inducing more hyperinflation and less severe reduction in $\mathrm{FEV} 1 .{ }^{28} \mathrm{In}$ fact, this possibility has been previously documented in patients with early COPD who showed emphysema and resting hyperinflation but normal expiratory flows. Furthermore, O'Donnell et al. clearly demonstrated that a volume effect can be induced with bronchodilators (i.e. reduction in operating lung volumes) with little or no impact on FEV1. Although it seems entirely plausible that there is no association between changes in FEV1 and lung volume, further studies are required before drawing conclusions on a specific sexbased susceptibility to hyperinflation. The functional impact of this different disease pattern in men and women was assessed using an incremental symptomslimited cycle exercise test. Although the interplay between airflow obstruction, lung volumes and exercise capacity is very complex ${ }^{28}$ when expressed in percentage of predicted value, women and men had a similar peak $\bullet \mathrm{VO} 2$. Although this would suggest similar disability between genders, the lower absolute $\cdot \mathrm{VO} 2$ value in women indicates a lower exercise reserve, and women may indeed be at a disadvantage when performing activities of daily living.

\section{Men and osteoporosis}

In Europe, osteoporosis causes vertebral, forearm or femoral fractures in approximately $46 \%$ of women and $22 \%$ of men after the age of 50 years. ${ }^{33}$ Male osteoporosis is a condition that is relatively unknown to many doctors. Currently in the US there are 500,000 fractures per year in males; in comparison, each year 200,000 men are diagnosed with prostate cancers. The mortality in the first year after the fracture is greater than $30 \%$ and approximately $50 \%$ of patients do not recover the mobility and independence they had before the event. The osteoporotic vertebral or femoral fractures in males generally have a worse prognosis compared to the same fractures in osteoporotic women of the same age. Canadian data show that the eco- 
nomic burden for the treatment and rehabilitation of osteoporotic fractures in males is approximately 70 million US dollars per year.

Again, prostatic cancer is the most common cancer in men: $50 \%$ of patients are expected to have androgen deprivation therapy (ADT), known to accelerate bone loss and increase the risk of fractures. Since 2006, Canadian experts recommend performing a test for bone mineral density (BMD) before ADT. Recent data suggest that, even among patients treated with ADT (at high risk for osteoporosis) or with a history of previous fragility fracture, dual-energy X-ray absorptiometry has been requested in less than $50 \%$ of cases. ${ }^{34-36}$

In males, the prevalence of secondary forms of osteoporosis is greater than in females (Table 1).

\section{Pathophysiology}

In males, a reduction in osteoblastic activity prevails, whereas an increased osteoclastic activity is seen in women. Consequently, the male osteoporotic bone quality is better than that of osteoporotic women. The peak bone mass is greater in the male compared to the female and this is reached later (16-17 years of age $v s$ 13-14 years). When the peak has been reached, both men and women begin to lose bone and this is related to the age-dependent decrease in gonadal steroids.

Table 1. Risk factors for male osteoporosis.

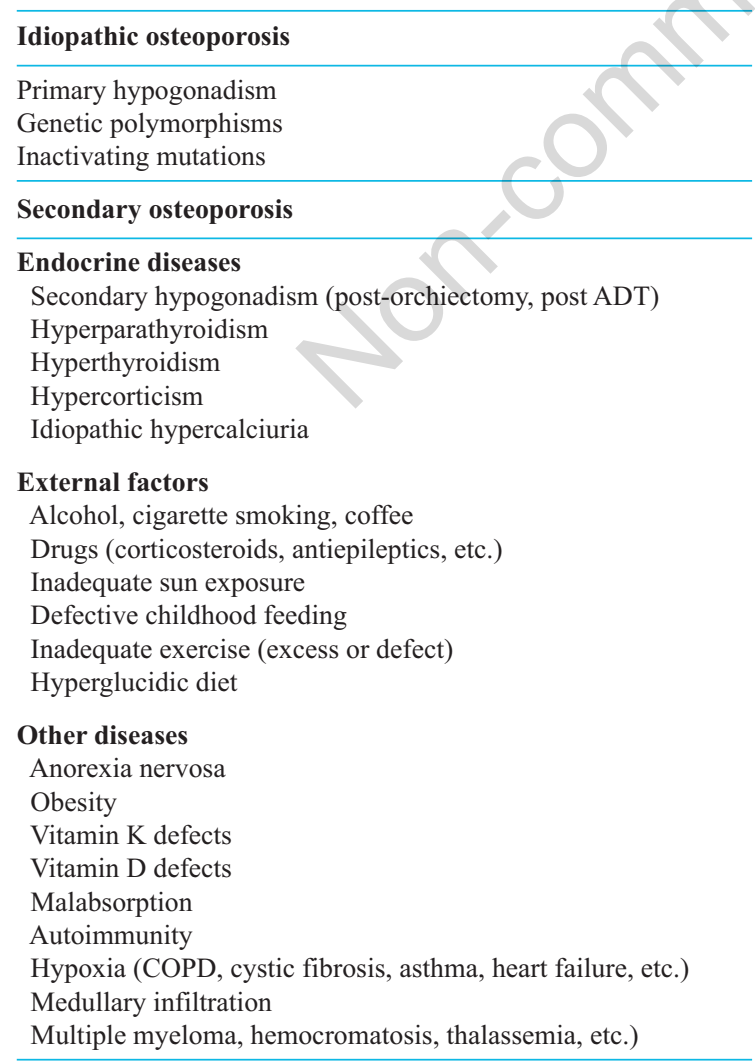

$\mathrm{ADT}$, androgen deprivation therapy; COPD, chronic obstructive pulmonary disease.
Testosterone and estrogen decrease over time asymmetrically. Estrogens levels in men are 10 times lower than in pre-menopausal women; while estrogens in senile males are double that of women. This is due to the rapid and severe menopause induced decrease in estrogen and also to the lower compensation in females. In fact, in females, the androgens that can be converted into estrogens derive exclusively from the adrenal gland, while in males the testes continue to work. Only in hypogonadic males are androgens and estrogens significantly reduced, and the trend of the BMD is similar in women. Among hypogonadic males, the onset of an eating disorder (anorexia nervosa) during adolescence has been recognized as an important cause of development of a more severe osteopenia/osteoporosis than in females. ${ }^{37}$

As we have already reported, peak bone mass is reached later in males than the females. Therefore, at the age of greatest risk for the onset of an eating disorder (13-15 years), the girls may have already reached their peak bone mass, while boys have a lower peak and will not reach normal stature. In a study of 70 males with anorexia nervosa, a higher prevalence of osteopenia and osteoporosis has been documented compared with a similar number of females with anorexia nervosa. A low body mass index (BMI) and longer disease duration were the best predictors of bone damage in these subjects. ${ }^{38}$

\section{Secondary forms}

Depression is an important contributing cause of male osteoporosis. In a recent study, ${ }^{39}$ depression, defined by a score greater than 6 on the Geriatric Depression Scale, is associated with an increased rate of femoral bone mass loss in a cohort of elderly males.

Numerous studies have shown an increased prevalence of osteopenia and osteoporosis in patients with COPD, who are mostly male. Literature data report osteoporosis is present in $20-32 \%$ of patients with COPD compared to $3-12 \%$ of controls, while osteopenia is present in more than $60 \%$ of them. Cigarette smoking, disease severity [according to the Global initiative for chronic Obstructive Lung Disease (GOLD) guidelines], nutritional status (BMI), hypogonadism, vitamin $\mathrm{D}$ deficiency and treatment with systemic steroids are considered risk factors for the development of fractures in patients with COPD. Even the use of topical steroids may increase the risk of fractures in these patients, although this occurs very rarely and is dose dependent. ${ }^{40}$

All men over the age of 70 years and those at increased risk of osteoporosis and fractures should be submitted to the following tests: i) height control: if a reduction of $3.5 \mathrm{~cm}$ is found, a spine X-ray is mandatory and compression fractures need to be excluded; ii) assessment of the risk of falling; iii) measurement 
of BMD: if T-score is in the range of osteoporosis, it is necessary to exclude secondary causes of low BMD.

In males after the age of 50 years, use the T-score of BMD in the report (the WHO classification criteria are applicable also in males). Before the age of 50 years, it is better to use the Z-Score (especially those under 20 years of age). A Z-Score lower of -2 is defined as below the expected range for the age, while if it is over the -2, BMD is defined as within the expected range for the age. In any case, the diagnosis of osteoporosis in men, before the age of 50 years, cannot be made solely on the basis of BMD.

\section{Therapy in men}

Therapy in men should be started on evidence of grade $\mathrm{A}$ for vitamin $\mathrm{D}$ and bisphosphonates, grade $\mathrm{C}$ for calcium, grade $\mathrm{D}$ for teriparatide.

\section{Male osteoporosis: clinical characteristics}

The clinical characteristics of male osteoporosis include: i) peak bone mass density reached at the time of skeletal maturity; ii) larger bone; iii) best-preserved bone architecture; iv) location on the wrist six times more frequent in men; v) later decrease of bone mass during lifespan; vi) increased prevalence of secondary forms; vii) higher mortality during rehabilitation after fracture; viii) lower functional recovery after fracture; ix) longer hospital stay after fracture; $x$ ) prevalence of trochanteric femoral fractures; xi) more comorbidities (pneumonia, sepsis, etc.); xii) higher mortality; xiii) greater fragility; xiv) vertebral fractures: increased prevalence of symptoms other than pain (poor quality of sleep, loss of energy, emotional difficulties, etc.).

\section{References}

1. Franconi F, Montilla S, Vella S, eds. Farmacologia di Genere. Torino: Ed SEEd; 2010.

2. European Society of Cardiology. Cardiovascular disease prevention - risk assessment and management. EuroPRevent 2013. Available from: http://www.escardio.org

3. Hermann HC, Klapp BF, Danzer G, Papachristou C. Gender-specific differences associated with living donor liver transplantation: a review study. Liver Transpl 2010;16:375-86.

4. Wizemann TM, Pardue ML (eds). Exploring the biological contributions to human health: Does sex matter? Washington, DC: National Academy of Sciences; 2001.

5. Franconi F, Carru C, Malorni W, et al. The effect of sex/gender on cardiovascular pharmacology. Curr Pharm Des 2011;17:1095-107.

6. Stramba-Badiale M. Women and research on cardiovascular diseases in Europe: a report from the European Heart Health Strategy (EuroHeart) project. Eur Heart J 2010;31:1677-81.

7. Levin RI. The puzzle of aspirin and sex. N Engl J Med 2005;352:1366-8.
8. Mosca L, Benjamin EJ, Berra K, et al. Effectivenessbased guidelines for the prevention of cardiovascular disease in women - 2011 update: a guideline from the American Heart Association. Circulation 2011;123: 1243-62.

9. Shaw LJ, Bugiardini R, Merz CN. Women and ischemic heart disease: evolving knowledge. J Am Coll Cardiol 2009;54:1561-75.

10. Kolovou G, Marvaki A, Bilianou H. One more look at guidelines for primary and secondary prevention of cardiovascular disease in women. Arch Med Sci 2011;7: 747-55.

11. Petrea RE, Beiser AS, Seshadri S, et al. Gender differences in stroke incidence and poststroke disability in the Framingham heart study. Stroke 2009;40:1032-7.

12. Istituto Superiore di Sanità. Registro per gli eventi coronarici e cerebrovascolari. Rapporti ISTISAN 2001. Roma: Istituto Superiore di Sanità; 2001. Available from: http://www.iss.it

13. Paolucci S, Bragoni M, Coiro P, et al. Is sex a prognostic factor in stroke rehabilitation? A matched comparison. Stroke 2006;37:2989-94.

14. Go AS, Mozaffarian D, Roger VL, et al. Heart disease and stroke statistics - 2013 update: a report from the American Heart Association. Circulation 2013;127:e6e245.

15. DeVon HA, Saban KL, Garrett DK. Recognizing and responding to symptoms of acute coronary syndromes and stroke in women. J Obstet Gynecol Neonatal Nurs 2011;40:372-82.

16. Antillon D, Towfighi A. No time to 'weight': the link between obesity and stroke in women. Womens Health (Lond Engl) 2011;7:453-63.

17. Ohayon MM, Priest RG, Guilleminault C, Caulet M. The prevalence of depressive disorders in the United Kingdom. Biol Psychiatry 1999;45:300-7.

18. Roy T, Lloyd CE. Epidemiology of depression and diabetes: a systematic review. J Affect Disord 2012;142: S8-21.

19. American Psychiatric Association. Diagnostic and statistical manual of mental disorders: DSM-IV. 4th ed. Washington (DC): American Psychiatric Association; 2000. Available from: http://www.psychiatry.org

20. La Carrubba S, Manna L, Rinollo C, et al. Depressive symptoms and disability in acute patients with comorbidities in departments of internal medicine. Ital J Med 2011;5:261-8.

21. Toohey J. Depression during pregnancy and postpartum. Clin Obstet Gynecol 2012;55:788-97.

22. Bottino CM, Barcelos-Ferreira R, Ribeiz SR. Treatment of depression in older adults. Curr Psychiatry Rep 2012;14:289-97.

23. Celli B, Vestbo J, Jenkins CR, et al. Sex differences in mortality and clinical expressions of patients with chronic obstructive pulmonary disease. The TORCH experience. Am J Respir Crit Care Med 2011;183:317-22.

24. Connett JE, Murray RP, Buist AS, et al. Changes in smoking status affect women more than men: results of the Lung Health Study. Am J Epidemiol 2003;157:973-9.

25. Cote CG, Chapman KR. Diagnosis and treatment considerations for women with COPD. Int J Clin Pract 2009;63:486-93.

26. Martinez FJ, Curtis J, Sciurba F, et al. Sex differences 
in severe pulmonary emphysema. Am J Respir Crit Care Med 2007;176:243-52.

27. Leynaert B, Bousquet J, Henry C, et al. Is bronchial hyperresponsiveness more frequent in women than in men? A population-based study. Am J Respir Crit Care Med 1997;156:1413-20.

28. Watson L, Vonk JM, Löfdahl CG, et al. Predictors of lung function and its decline in mild to moderate COPD in association with gender: results from the Euroscop study. Respir Med 2006;100:746-53.

29. Canadian Institute for Health Information; Canadian Lung Association; Health Canada; Statistics Canada. Respiratory disease in Canada. Ottawa, ON: Publich Health Agency of Canada; 2001. Available from: http://www.phac-aspc.gc.ca

30. Liu S, Zhou Y, Wang X, et al. Biomass fuels are the probable risk factor for chronic obstructive pulmonary disease in rural South China. Thorax 2007;62:889-97.

31. Sørheim IC, Johannessen A, Gulsvik A, et al. Gender differences in COPD. Thorax 2010;65:480-5.

32. de Torres JP, Casanova C, de Garcini AM, et al. COPD heterogeneity: gender differences in the multidimensional BODE index. Int J Chron Obstruct Pulmon Dis 2007;2:151-5.
33. Hasserius R, Karlsson MK, Jónsson B, et al. Long-term morbidity and mortality after a clinically diagnosed vertebral fracture in the elderly: a 12 and 22-year followup of 257 patients. Calcif Tissue Int 2005;76:235-42.

34. Gruntmanis U. Male osteoporosis: deadly, but ignored. Am J Med Sci 2007;333:85-92.

35. Tarride JE, Guo N, Hopkins R, et al. The burden of illness of osteoporosis in Canadian men. J Bone Miner Res 2012;27:1830-8.

36. Center JR, Nguyen TV, Schneider D, et al. Mortality after all major types of osteoporotic fracture in men and women: an observational study. Lancet 1999;353:878-82.

37. Mehler PS, Sabel AL, Watson T, Andersen AE. High risk of osteoporosis in male patients with eating disorders. Int J Eat Disord 2008;41:666-72.

38. Diem SJ, Harrison SL, Haney E, et al. Depressive symptoms and rates of bone loss at the hip in older me. Osteoporosis Int 2012;24:111-9.

39. Herrera A, Lobo-Escolar A, Mateo J, et al. Male osteoporosis: a review. World J Orthop 2012;3:223-34.

40. Vrieze A, de Greef MH, Wijkstra PJ, Wempe JB. Low bone mineral density in COPD patients related to worse lung function, low weight and decreased fat-free mass. Osteoporos Int 2007;18:1197-202. 